\title{
Prognostic markers in patients who have recovered from an acute variceal bleeding: role of HVPG measurement
}

\author{
Rafael Bañares*, María-Vega Catalina, Cristina Ripoll and Diego Rincón \\ Liver and Transplant Unit. Instituto de Investigación Sanitaria Gregorio Marañón, Ciberehd, Madrid, Spain
}

\section{Introduction}

Variceal bleeding is one of the most severe complications of cirrhosis which despite recent advances in its management, still carries a high mortality. This situation is especially important in those patients surviving a first episode of variceal bleeding who are at higher risk of recurrent bleeding with an estimated $60 \%$ risk within one year after the first bleeding episode. Therefore, prevention of rebleeding remains a very important issue in the management of variceal bleeding. However, the risk of rebleeding is not homogeneous through the overall spectrum of bleeding patients, suggesting the need to stratify the rebleeding risk and to adjust the intensity of therapy according to this risk. In this manuscript we will review the utility of HVPG measurement in variceal rebleeding and its potential impact in tailoring therapy.

\section{Critical analysis of the evidence supporting the role of HVPG measurement in secondary prophylaxis}

Randomized clinical trials have shown that longterm therapy with pharmacological agents reduces the risk of recurrent variceal bleeding by $40 \%$ [1]. However it is assumed that to confer protection, drug therapy should achieve a target reduction in portal pressure usu-

${ }^{*}$ Corresponding author: Liver and Transplant Unit. Instituto de Investigación Sanitaria Gregorio Marañón. Ciberehd, Universidad Complutense, Madrid, Spain. E-mail: rbanares@telefonica.net. ally estimated by sequential measurements of hepatic venous pressure gradient (HVPG). Importantly, HVPG measurements should be performed according to welldefined technical conditions aimed to provide accurate and valid measurements [2].

An ancillary longitudinal pharmacological study in primary prophylaxis of variceal bleeding demonstrated that the risk of bleeding was zero when the HVPG decreased below a threshold value of $12 \mathrm{mmHg}$ [3]. Subsequent studies showed that, even without reaching this target, the residual risk of bleeding is low when drug therapy achieves an HVPG reduction of $>20 \%$ from baseline although this finding has been questioned in other studies [4,5], especially in secondary prophylaxis. However, two recent systematic reviews with metaanalysis $[6,7]$ have confirmed the ability of HVPG reduction to predict the risk of rebleeding and death. Both meta-analysis reach similar conclusions that in summary are:1) a complete HVPG response (as defined as a final HVPG value less than $12 \mathrm{mmHg}$ ) is associated to an almost complete removal of the rebleeding risk; 2) a partial response (as defined as a HVPG reduction of more than $20 \%$ from baseline) is also associated with a significant reduction in the risk of rebleeding when an outlier trial was excluded (see below); 3) achieving a hemodynamic response conferred not only protection against variceal bleeding, but also a significant reduction in liver-related mortality. Importantly, both metaanalysis incorporated in their design robust statistical tools to detect and analyze heterogeneity that is almost uniformly associated to meta-analysis of observational studies. Meta-regression analysis identified that the main source of heterogeneity in the predictive ability of HVPG was the time interval between the two hemody- 
namic measurements (the longer the interval the lower the ability of HVPG to predict the risk of rebleeding). According to these data, the exclusion of an outlier trial due to the prolonged interval between the first and the second hemodynamic study, almost completely abrogated heterogeneity. This is an important observation because the probability of bleeding recurrence is greater immediately after the index hemorrhage and therefore the delay between hemodynamic studies may lead to bias in the risk prediction according to HVPG response. On the other hand, the exclusion of patients throughout the different studies because of rebleeding before the scheduled repeat measurement could also lead to bias. In order to evaluate this aspect, one of the meta-analysis [7] checked the robustness of the estimate by considering that all non-evaluable patients in the studies were from the worst-case scenario, which is HVPG responders and bleeders. Even in this setting, the bleeding risk was still significantly lower in overall responders, suggesting the robustness of the estimation.

Unfortunately, different factors that have been implicated in HVPG response such as the degree of liver failure, the dose of drugs, the extent of portocollateral shunt and beta-2-adrenoceptor gene polymorphisms, cannot be used as predictors of response due to its lack of predictive ability [8,9]. Similarly, it is well established that non-invasive parameters such as heart rate decrease or ultrasonographic parameters do not predict hemodynamic response.

Nevertheless, the clinical application of routine HVPG measurements is hampered by the need of repeat invasive procedures that is demanding and costly, and by the fact that, as previously commented, a significant number of patients might develop bleeding before the second hemodynamic study. In this setting, two recent studies have assessed the potential predictive ability of the acute HVPG response to intravenous bolus administration of propranolol at the baseline hemodynamic study $[10,11]$. In one of these studies [10], a subanalysis in previous bleeders was performed. Interestingly, a $12 \%$ reduction of HVPG from baseline values at 20 minutes after a bolus of intravenous propranolol was associated with an adequate prediction of the risk of rebleeding. The actuarial probability of developing a portal hypertension-related bleeding was two-fold greater in non-responders (as defined by the $12 \%$ cutoff) than in responders $(31 \%$ and $46 \%$ at 1 and 2 years vs. $17 \%$ and $23 \%$, respectively). Importantly, the usual HVPG reduction used in the assessment of chronic response (20\% reduction from baseline) was not associated to accurate prognostic information. Interestingly, on mul- tivariate analysis only HVPG response and previous history of variceal bleeding were associated to the rebleeding risk. Moreover, the combination of these two independent variables identified a very high-risk population. Therefore, patients without acute HVPG response to propranolol and a history of more than one previous bleeding episode showed the highest risk of rebleeding during follow-up ( $78 \%$ at 2 year), clearly greater than the rate observed in patients with only one of these risk factors (isolated non-responders: $35 \%$ at two years; more than one bleeding episode in hemodynamic responders: $25 \%$ at two years). These results suggest several important considerations. First, it is possible to evaluate chronic response to drug therapy in patients with a past history of bleeding during a single hemodynamic study, thus avoiding the need of a second study and simplifying the evaluation of the hemodynamic response. Second, the use of less stringent criteria in the evaluation of HVPG acute response as compared with the chronic HVPG response, might reduce what has been called the "grey zone" of response (patients that despite being non-responders have a good clinical outcome), thus expanding the clinical application of HVPG measurement. Third, the combination of both independent predictive variables (HVPG response and more than one previous episode of bleeding) can discriminate a population of patients with a very high risk of rebleeding in whom an early decision for alternative therapy may be justified.

Further studies are required to validate the usefulness of acute evaluation of HVPG response in the evaluation of hemodynamic response in patients with variceal bleeding

\section{Potential clinical utility of risk stratification (design of "à la carte" therapies)}

Taking into account the strong predictive ability of HVPG measurements, several studies have analyzed its use as a tool to individualize clinical management of these patients. This concept is especially important considering the existence of several therapeutic alternatives (multiple drug therapy, band ligation, TIPS, surgery and liver transplantation) with different risks, applicability, costs, etc. In the acute setting, the use of a risk stratification based approach has proved to be successful tool in patients with acute variceal bleeding. In this context, an aggressive initial management with TIPS placement in high-risk patients is associated to a survival improvement [12]. 
The efficacy of non-selective beta-blockers in the secondary prophylaxis of variceal bleeding has been proven in many trials and these drugs are widely accepted as the first-line pharmacological therapy [13]. Non-selective beta-blockers reduce the risk of rebleeding [1], mortality and rebleeding-related mortality [14]. Although the addition of mononitrate isosorbide enhanced the portal pressure decreasing effect of betablockers [15], the clinical efficacy of this combination has not been clearly shown. The first study that assessed tailored therapy according to this concept was developed by Bureau et al. [16]. As a part of this study, 14 patients with previous variceal bleeding received a first hemodynamic study before starting therapy with a fix-dose of long-acting propranolol. In patients without an adequate hemodynamic response in a second study, isosorbide mononitrate was added and the hemodynamic response evaluated in a third hemodynamic study. Non-responding patients were treated by adding band ligation. The main result of the study was the confirmation of the strong relationship between HVPG response and clinical events during follow-up and the identification of a higher risk population amenable to receive more aggressive therapy. Unfortunately, the small sample size precluded the clinical applicability of these conclusions.

The combination of beta-blockers with prazosin caused a greater decrease in portal pressure than betablockers alone $[17,18]$, increasing the proportion of hemodynamic responders to over $80 \%$. According to these relevant hemodynamic finding a recent trial has examined the usefulness of a HVPG guided therapy with betablockers plus prazosin [19]. In this study 59 patients were randomized to receive combined therapy with nadolol plus endoscopic variceal ligation vs. pharmacological therapy tailored according HVPG monitoring. Patients randomized to pharmacological therapy received combined nadolol plus isosorbide mononitrate until a second hemodynamic study was performed. In those patients without hemodynamic response (as previously defined) isosorbide mononitrate was substituted to carefully titrated prazosin and received a third hemodynamic study to assess the final response. The main results of the study were 1) the use of a HVPG guided approach was able to induce an overall hemodynamic response in $74 \%$ of patients. Importantly, further hemodynamic response was observed in 10 out of 13 patients on prazosin 2) the probability of rebleeding was very low in responders, independently of the group of randomization $(14 \%$ in patients randomized to nadolol+EVL and $15 \%$ in patients randomized to
HVPG guided drug therapy) 3) The use of HVPG guided therapy was not associated to significant worsening of systemic hemodynamics, increase of ascites or edema. However, drug-related adverse events (weakness, dizziness, headaches or impotence) were more frequent in HVPG guided group. 4) The improvement in the proportion of patients with hemodynamic response in the HVPG-guided therapy group did not translate to a reduction in the rate of recurrent bleeding, probably due to the assumptions used to calculate sample size based on the probability to reach hemodynamic response. Unfortunately the clinical use of this approach is clearly hampered by the need to perform up to three hemodynamic studies.

In another study a multistep approach based in HVPG measurements was used to tailoring therapy [20], examining several approaches with different degrees of aggressiveness. In this study 50 patients received a first hemodynamic study at day five after the index bleeding, starting immediately with combined betablockers and nitrates therapy, and then performing a second study 5-7 days after obtained maximum doses of drugs. Eight patients could not receive a second HVPG measurement, mainly due to early rebleeding. Patients were then classified according its response in three groups: hemodynamic responders (as previously defined; 57\%), partial responders (HVPG decrease between $10-20 \%$ from baseline value; $24 \%$ )) and no responders (HVPG decrease less than $10 \%$ from baseline value; $19 \%$ ). After the hemodynamic assessments, patients were treated according to their hemodynamic response: complete responders were maintained on drug therapy, partial responders were treated with additional band ligation and non responders received TIPS insertion (with PTFE-coated prostheses in the majority of cases). The end-point of the study was the incidence of clinically significant bleeding. The overall incidence of rebleeding was $22 \%$ at 2 years but only $12 \%$ of patients in whom hemodynamic response could be assessed. Additionally there were no differences among the three groups (12\% in drug therapy group, $20 \%$ in banding plus drugs group and $0 \%$ in TIPS group). The main conclusion of this open-label study is that a hemodynamic response-guided therapy for prevention of rebleeding is feasible and might provide an effective strategy in individualization of therapy. However and as previously commented, the proposed hemodynamic evaluation is highly demanding, and unfortunately, the study included a small number of patients.

Further insight in the possible utility of HVPG assessment comes from a recent study aimed to compare 
combined drug therapy with combined drug therapy plus variceal band ligation. In this study the authors found no significant differences in rebleeding rates in HVPG non-responders treated with drugs alone or with drugs plus variceal ligation, suggesting that adding ligation may not be the best alternative to reduce rebleeding in HVPG non-responders [21] and that perhaps a more aggressive approach is recommended in such high-risk patients.

All these studies lead to two main conclusions or hypothesis: The first one is that to obtain hemodynamic response should be the goal of any therapeutic alternative, and therefore the ideal therapy in the prevention of rebleeding would be one able to obtain hemodynamic response in the majority of cases (avoiding the need to perform hemodynamic studies), free of adverse events and easy to administer. Several drugs or drugs combination have been suggested in this context as carvedilol [22-24], angiotensin inhibitors [25] or simvastatin [26]. Unfortunately none of them has been appropriately tested in the context of prevention of rebleeding. Importantly, the possibility to obtain accurate surrogate markers of hemodynamic response able to avoid aggressive hemodynamic measurements should be considered as an important research question in that field.

The second is that hemodynamic non-responding patients have an increase risk of rebleeding and therefore, could benefit from more aggressive approaches such TIPS in selected patients, although this hypothesis should be tested in appropriate clinical trials.

\section{References}

[1] G. D'Amico, L. Pagliaro and J. Bosch, Pharmacological treatment of portal hypertension: an evidence-based approach, Semin Liver Dis 19 (1999), 475-505.

[2] R.J. Groszmann and S. Wongcharatrawee, The hepatic venous pressure gradient: anything worth doing should be done right, Hepatology 39 (2004), 280-282.

[3] R.J. Groszmann, J. Bosch, N.D. Grace, H.O. Conn, G. GarciaTsao, M. Navasa, J. Alberts et al., Hemodynamic events in a prospective randomized trial of propranolol versus placebo in the prevention of a first variceal hemorrhage, Gastroenterology 99 (1990), 1401-1407.

[4] U. Thalheimer, M. Mela, D. Patch and A.K. Burroughs, Targeting portal pressure measurements: a critical reappraisal, Hepatology 39 (2004), 286-290.

[5] U. Thalheimer, M. Mela, D. Patch and A.K. Burroughs, Monitoring target reduction in hepatic venous pressure gradient during pharmacological therapy of portal hypertension: a close look at the evidence, Gut 53 (2004), 143-148.

[6] G. D'Amico, J.C. Garcia-Pagan, A. Luca and J. Bosch, Hepatic vein pressure gradient reduction and prevention of variceal bleeding in cirrhosis: a systematic review, Gastroenterology 131 (2006), 1611-1624.
[7] A. Albillos, R. Banares, M. Gonzalez, C. Ripoll, R. Gonzalez, M.V. Catalina and L.M. Molinero, Value of the hepatic venous pressure gradient to monitor drug therapy for portal hypertension: a meta-analysis, Am J Gastroenterol 102 (2007), 1116-1126.

[8] J. Turnes, M. Hernandez-Guerra, J.G. Abraldes, P. Bellot, R. Oliva, J.C. Garcia-Pagan and J. Bosch, Influence of beta-2 adrenergic receptor gene polymorphism on the hemodynamic response to propranolol in patients with cirrhosis, Hepatology 43 (2006), 34-41.

[9] A. Escorsell, J.M. Bordas, B. Castaneda, J. Llach, J.C. GarciaPagan, J. Rodes and J. Bosch, Predictive value of the variceal pressure response to continued pharmacological therapy in patients with cirrhosis and portal hypertension, Hepatology 31 (2000), 1061-1067.

[10] V. La Mura, J.G. Abraldes, S. Raffa, O. Retto, A. Berzigotti, J.C. Garcia-Pagan and J. Bosch, Prognostic value of acute hemodynamic response to i.v. propranolol in patients with cirrhosis and portal hypertension, J Hepatol 51 (2009), 279287.

[11] C. Villanueva, C. Aracil, A. Colomo, V. Hernandez-Gea, J.M. Lopez-Balaguer, C. Alvarez-Urturi, X. Torras et al., Acute hemodynamic response to beta-blockers and prediction of long-term outcome in primary prophylaxis of variceal bleeding, Gastroenterology 137 (2009), 119-128.

[12] J.C. Garcia-Pagan, K. Caca, C. Bureau, W. Laleman, B. Appenrodt, A. Luca, J.G. Abraldes et al., Early use of TIPS in patients with cirrhosis and variceal bleeding, $N$ Engl $J$ Med 362 (2010), 2370-2379.

[13] R. de Franchis, Evolving consensus in portal hypertension. Report of the Baveno IV consensus workshop on methodology of diagnosis and therapy in portal hypertension, J Hepatol $\mathbf{4 3}$ (2005), 167-176.

[14] B. Bernard, D. Lebrec, P. Mathurin, P. Opolon and T. Poynard, Beta-adrenergic antagonists in the prevention of gastrointestinal rebleeding in patients with cirrhosis: a meta-analysis, Hepatology 25 (1997), 63-70.

[15] J.C. Garcia-Pagan, F. Feu, J. Bosch and J. Rodes, Propranolol compared with propranolol plus isosorbide-5-mononitrate for portal hypertension in cirrhosis. A randomized controlled study, Ann Intern Med 114 (1991), 869-873.

[16] C. Bureau, J.M. Peron, L. Alric, J. Morales, J. Sanchez, K. Barange, J.L. Payen et al., "A La Carte" treatment of portal hypertension: Adapting medical therapy to hemodynamic response for the prevention of bleeding, Hepatology 36 (2002), 1361-1366.

[17] A. Albillos, J.L. Lledo, R. Banares, I. Rossi, J. Iborra, J.L. Calleja, A. Garrido et al., Hemodynamic effects of alphaadrenergic blockade with prazosin in cirrhotic patients with portal hypertension, Hepatology 20 (1994), 611-617.

[18] A. Albillos, J.C. Garcia-Pagan, J. Iborra, J.C. Bandi, G. Cacho, M. Perez-Paramo, A. Escorsell et al., Propranolol plus prazosin compared with propranolol plus isosorbide-5- mononitrate in the treatment of portal hypertension, Gastroenterology 115 (1998), 116-123.

[19] C. Villanueva, C. Aracil, A. Colomo, J.M. Lopez-Balaguer, M. Piqueras, B. Gonzalez, X. Torras et al., Clinical trial: a randomized controlled study on prevention of variceal rebleeding comparing nadolol + ligation vs. hepatic venous pressure gradient-guided pharmacological therapy, Aliment Pharmacol Ther 29 (2009), 397-408.

[20] A. Gonzalez, S. Augustin, M. Perez, J. Dot, E. Saperas, A. Tomasello, A. Segarra et al., Hemodynamic response-guided 
therapy for prevention of variceal rebleeding: an uncontrolled pilot study, Hepatology 44 (2006), 806-812.

[21] J.C. Garcia-Pagan, C. Villanueva, A. Albillos, R. Banares, R. Morillas, J.G. Abraldes and J. Bosch, Nadolol plus isosorbide mononitrate alone or associated with band ligation in the prevention of recurrent bleeding: a multicentre randomised controlled trial, Gut 58 (2009), 1144-1150.

[22] R. Banares, E. Moitinho, B. Piqueras, M. Casado, J.C. GarciaPagan, A. de Diego and J. Bosch, Carvedilol, a new nonselective beta-blocker with intrinsic anti- Alpha1-adrenergic activity, has a greater portal hypotensive effect than propranolol in patients with cirrhosis, Hepatology 30 (1999), 79-83.

[23] R. Banares, E. Moitinho, A. Matilla, J.C. Garcia-Pagan, J.L. Lampreave, C. Piera, J.G. Abraldes et al., Randomized comparison of long-term carvedilol and propranolol administration in the treatment of portal hypertension in cirrhosis, Hep- atology 36 (2002), 1367-1373.

[24] D. Tripathi, J.W. Ferguson, N. Kochar, J.A. Leithead, G. Therapondos, N.C. McAvoy, A.J. Stanley et al., Randomized controlled trial of carvedilol versus variceal band ligation for the prevention of the first variceal bleed, Hepatology 50 (2009), $825-833$.

[25] P. Tandon, J.G. Abraldes, A. Berzigotti, J.C. Garcia-Pagan and J. Bosch, Renin-angiotensin-aldosterone inhibitors in the reduction of portal pressure: a systematic review and metaanalysis, J Hepatol 53, 273-282.

[26] J.G. Abraldes, A. Albillos, R. Banares, J. Turnes, R. Gonzalez, J.C. Garcia-Pagan and J. Bosch, Simvastatin lowers portal pressure in patients with cirrhosis and portal hypertension: a randomized controlled trial, Gastroenterology 136 (2009), $1651-1658$. 


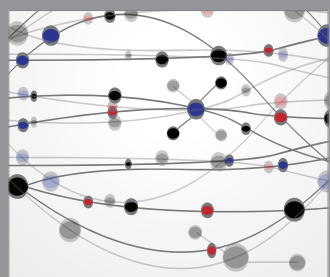

The Scientific World Journal
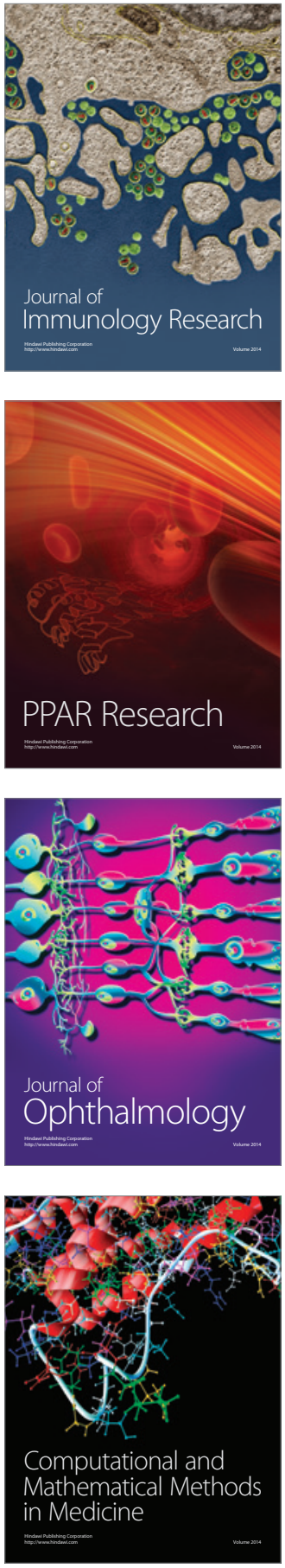

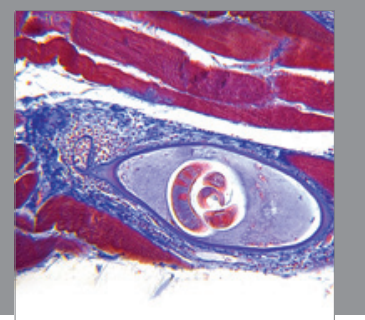

Gastroenterology

Research and Practice
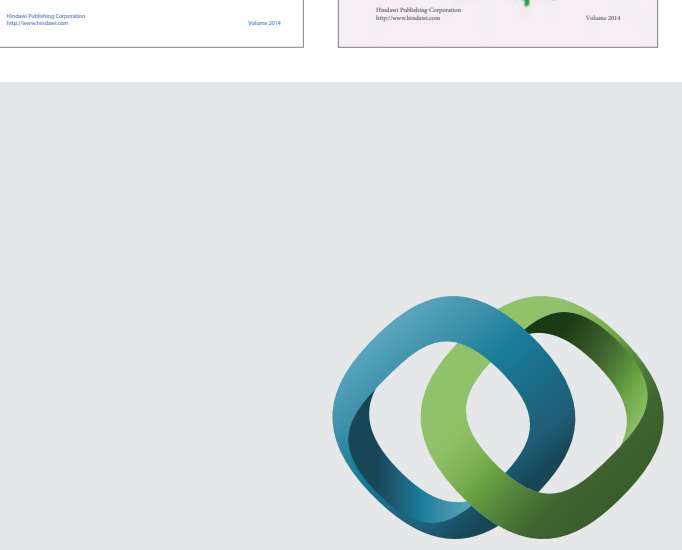

\section{Hindawi}

Submit your manuscripts at

http://www.hindawi.com
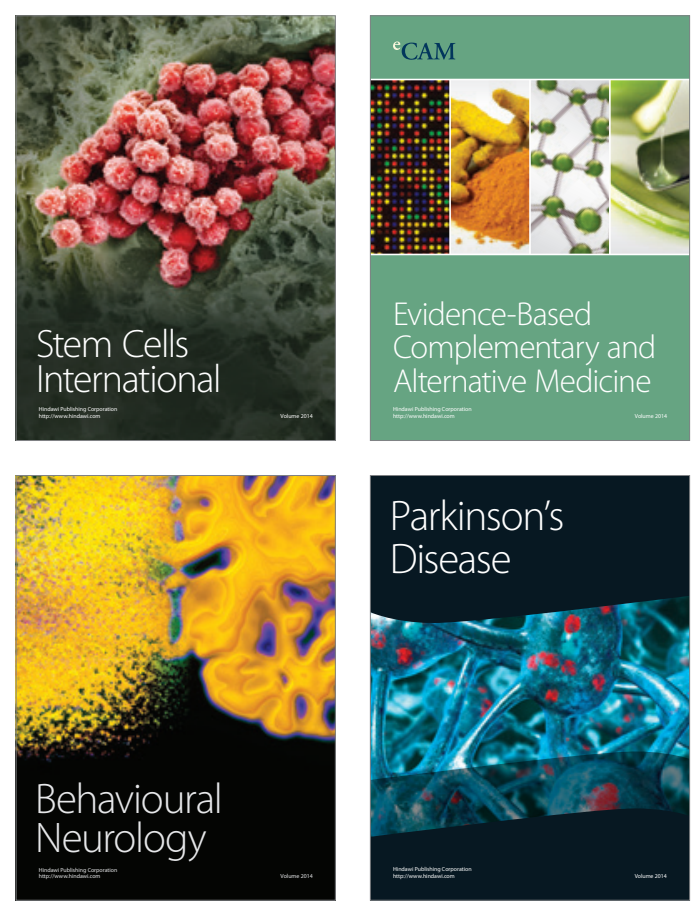

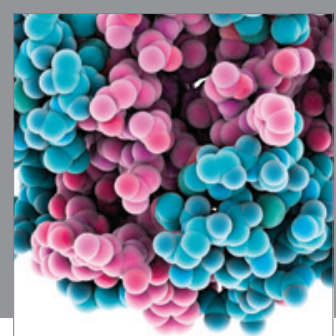

Journal of
Diabetes Research

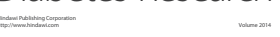

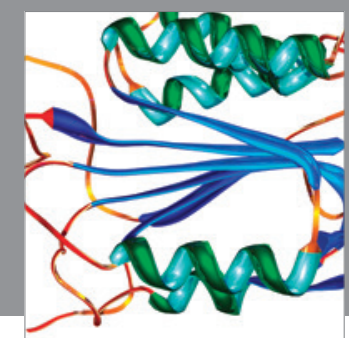

Disease Markers
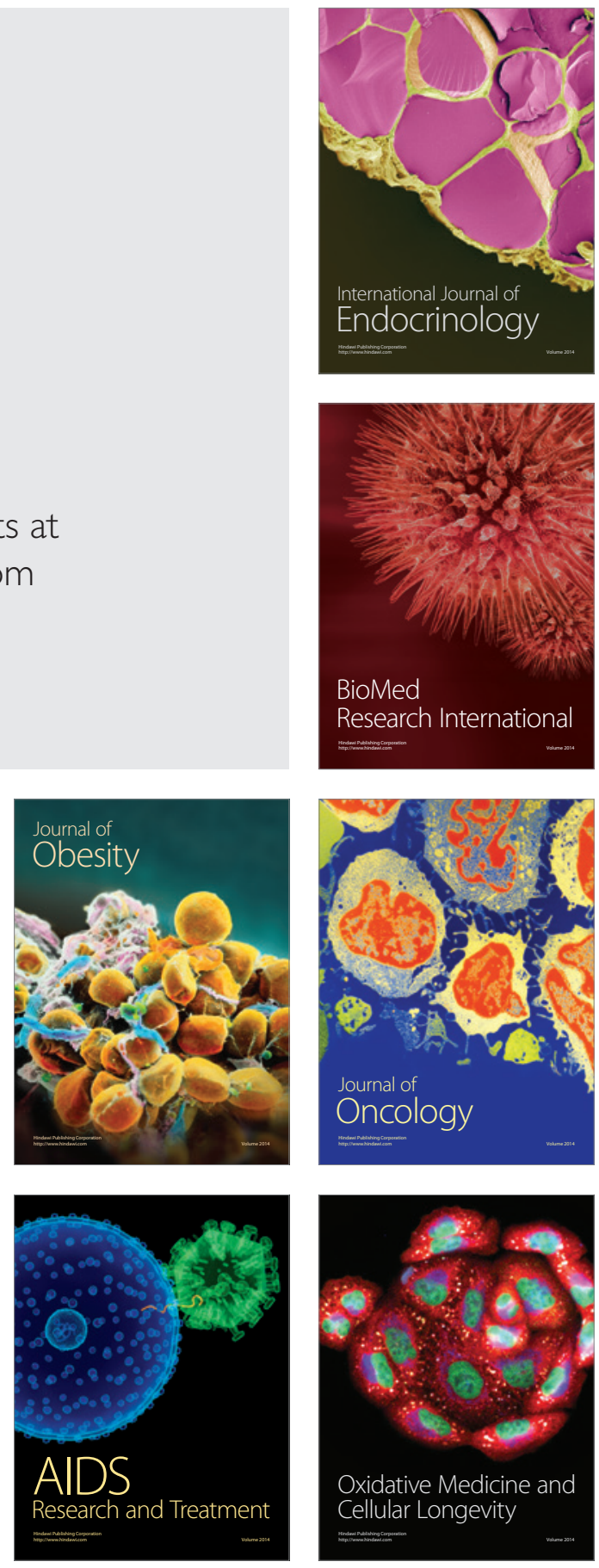